\title{
Dexamethasone and Long-Term Outcome in Adults with Bacterial Meningitis
}

\author{
Martijn Weisfelt, MD, ${ }^{1}$ Martine Hoogman, MSc, ${ }^{1}$ Diederik van de Beek, PhD, ${ }^{1}$ Jan de Gans, PhD, ${ }^{1}$ \\ Wouter A. Dreschler, $\mathrm{PhD},{ }^{2}$ and Ben A. Schmand, $\mathrm{PhD}^{1,3}$
}

Objective: This follow-up study of the European Dexamethasone Study was designed to examine the potential harmful effect of adjunctive dexamethasone treatment on long-term neuropsychological outcome in adults with bacterial meningitis.

Methods: Neurological, audiological, and neuropsychological examinations were performed in adults who survived pneumococcal or meningococcal meningitis.

Results: Eighty-seven of $99(88 \%)$ eligible patients were included in the follow-up study; 46 (53\%) were treated with dexamethasone and $41(47 \%)$ with placebo. Median time between meningitis and testing was 99 months. Neuropsychological evaluation showed no significant differences between patients treated with dexamethasone and placebo. The proportions of patients with persisting neurological sequelae or hearing loss were similar in the dexamethasone and placebo groups. The overall rate of cognitive dysfunction did not differ significantly between patients and control subjects; however, patients after pneumococcal meningitis had a higher rate of cognitive dysfunction $(21 \mathrm{vs} 6 \% ; p=0.05)$ and experienced more impairment of everyday functioning due to physical problems $(p=0.05)$ than those after meningococcal meningitis.

Interpretation: Treatment with adjunctive dexamethasone is not associated with an increased risk for long-term cognitive impairment. Adults who survive pneumococcal meningitis are at significant risk for long-term neuropsychological abnormalities.

Ann Neurol 2006;60:456-468

The estimated incidence of bacterial meningitis is 2 to 5 per 100,000 adults per year. ${ }^{1-3}$ The predominant causative pathogens in adults are Streptococcus pneumoniae and Neisseria meningidis, which cause 80 to $85 \%$ of all cases. ${ }^{1,4}$ Reported mortality rates are high $(20-30 \%)$, and neurological sequelae occur in a high proportion of surviving patients $(15-40 \%){ }^{1,4}$ Cognitive impairment occurs frequently after bacterial meningitis. ${ }^{2}$ In one prospective study, cognitive impairment was detected in $27 \%$ of adults who had a good recovery from pneumococcal meningitis. ${ }^{5}$ In this study, cognitive impairment consisted mainly of cognitive slowness, which was related to lower scores on questionnaires measuring the quality of life. Patients after meningococcal meningitis were not significantly different from control subjects. ${ }^{5}$

A European randomized, placebo-controlled trial involving 301 adults with suspected meningitis showed that adjunctive treatment with dexamethasone before or with the first dose of antimicrobial therapy reduced the risk for an unfavorable outcome from 25 to $15 \%{ }^{6}$ Mortality was reduced from 15 to $7 \%$. In a recent quantitative review of this topic, treatment with corti- costeroids was associated with a significant reduction in mortality and neurological sequelae. ${ }^{7}$ Since publications of these results, adjunctive dexamethasone has become routine therapy in adults with suspected bacterial meningitis. $^{2,6,8}$

Corticosteroids may potentiate ischemic and apoptotic injury to neurons.? In a model of pneumococcal meningitis in infant rats, adjunctive dexamethasone aggravated neuronal apoptosis in the hippocampal dentate compared with antibiotic therapy alone. ${ }^{10}$ Increased hippocampal cell injury caused by dexamethasone was related to a reduced learning capacity in this model. ${ }^{10}$ The effect of adjunctive dexamethasone therapy on cognitive outcome in humans after bacterial meningitis is unknown. , $^{2,11-14}$

This study was designed to examine the potential harmful effects of adjunctive dexamethasone treatment on long-term cognitive outcome in patients with bacterial meningitis. Therefore, neurological, audiological, and neuropsychological examinations were performed in adults included in the European Dexamethasone Study after pneumococcal or meningococcal meningitis.
From the ${ }^{1}$ Departments of Neurology and ${ }^{2}$ Audiology, Center of Infection and Immunity Amsterdam (CINIMA), Academic Medical Center; and ${ }^{3}$ Department of Psychology, University of Amsterdam, Amsterdam, the Netherlands.

Received Apr 10, 2006, and in revised form Jun 23. Accepted for publication Jul 5, 2006.
M.W. and M.H. contributed equally to this article.

Published online Sep 6, 2006, in Wiley InterScience (www.interscience.wiley.com). DOI: 10.1002/ana.20944

Address correspondence to Dr Weisfelt, Department of Neurology, Academic Medical Center, PO Box 22660, 1100 DD Amsterdam, the Netherlands. E-mail: m.weisfelt@amc.uva.nl 


\section{Subjects and Methods Initial Study}

The European Dexamethasone Study was a double-blind, placebo-controlled trial of adjunctive dexamethasone therapy for adults with bacterial meningitis. Eligible patients were older than 17 years and had suspected meningitis in combination with cloudy cerebrospinal fluid (CSF), bacteria in CSF on Gram staining, or a CSF leukocyte count greater than $1,000 / \mathrm{mm}^{3}{ }^{6}$ Patients were enrolled between June 1993 and December 2001. A total of 301 patients were randomly assigned to receive dexamethasone sodium phosphate, at a dose of $10 \mathrm{mg}$ given every 6 hours intravenously for 4 days, or placebo. The study medication was given 15 to $20 \mathrm{~min}$ utes before or with the first dose of antibiotics. The primary outcome measure was the score on the Glasgow Outcome Scale (GOS) 8 weeks after randomization, as assessed by the patient's physician. This is a well-validated measurement scale with a score range from 1 to 5: a score of 1 indicates death; 2 is persistent vegetative state; 3 is severe disability; 4 is moderate disability (disabled but independent); and 5 is good recovery (mild or no disability). ${ }^{15} \mathrm{~A}$ favorable outcome was defined as a score of 5 , and an unfavorable outcome as a score of 1 to 4. CSF culture yielded S. pneumoniae in 108 patients (36\%), N. meningitidis in 97 patients (32\%), and other bacteria in 31 patients (10\%); CSF cultures remained negative in 65 patients (22\%). Treatment with dexamethasone was associated with a reduction in the risk for an unfavorable outcome (relative risk, $0.6 ; 95 \%$ confidence interval, $0.3-0.9 ; p=0.03$ ) and mortality (relative risk, 0.48; $95 \%$ confidence interval, $0.2-0.96 ; p=0.04){ }^{6}$

\section{Follow-up Study}

Patients eligible for this follow-up study were older than 17 years living in the Netherlands who survived pneumococcal or meningococcal meningitis, confirmed by CSF culture. Exclusion criteria were serious illnesses other than meningitis interfering with cognitive testing, pre-existing psychiatric disorders, evidence for alcoholism or other substance abuse, use of sedatives or neuroleptic medication, and insufficient mastery of the Dutch language. Patients were recruited by contacting treating physicians, general practitioners, or through municipality databases. The study was approved by the ethics committee of the Academic Medical Center. For each of the participating patients, 1 control subject was recruited from partners, siblings, and close friends until a control group of 50 subjects was completed. All participants gave written, informed consent. Patients and control subjects underwent a tone and speech audiogram and a neuropsychological evaluation by a psychologist (M.H.) who was blinded with respect to the former treatment group (dexamethasone or placebo). Whenever audiometry was performed, hearing loss was expressed as the average degree of pure-tone hearing loss of high frequencies $(1,2$, and $4 \mathrm{kHz})$ on the most severely affected side. Hearing loss was classified as follows: normal $(<30 \mathrm{~dB})$, mild $(\geq 30-55 \mathrm{~dB})$, moderate $(\geq 55-70 \mathrm{~dB})$, severe $(\geq 70-90 \mathrm{~dB})$, or profound $(\geq 90 \mathrm{~dB}) .{ }^{16}$ Two experienced physicians (M.W. and D.v.d.B.) performed a neurological examination in patients. Focal neurological deficits were divided into focal cerebral deficits (aphasia, monoparesis, or hemiparesis) and cranial nerve palsies. Participants unable or unwilling to travel to our hospital underwent a neuropsychological evaluation by our psychologist in a hospital near the patients' residences.

\section{Neuropsychological Evaluation}

Six cognitive domains were tested by the following test battery:

1. Intelligence: Current intelligence was tested by the Groningen Intelligence Tests (GIT)-abbreviated version, consisting of subtests for verbal and visuospatial reasoning and numerical ability ${ }^{17}$; premorbid intelligence was tested by the Dutch Adult Reading Test. ${ }^{18}$

2. Memory: The Rey's Auditory Verbal Learning Test (AVLT) and the subtest Story Recall from the Rivermead Behavioural Memory Test (RBMT) were used to test verbal memory, ${ }^{19,20}$ visual memory was tested by the subtest Visual Reproduction from the Wechsler Memory Scale-Revised (WMS-R), ${ }^{19}$ and digit span was assessed by the Wechsler Adult Intelligence ScaleRevised (WAIS-R) Digit Span Test. ${ }^{21}$

3. Language: A short form of the Boston Naming Test (BNT) was used to examine language function; the score was extrapolated to the full-length score. ${ }^{22}$

4. Attention: Attention was assessed by the Trail Making Test part B, Stroop Color Word Test part C (interference condition), and the Wechsler Adult Intelligence Scale-Revised Digit Span Test. ${ }^{19,21}$

5. Executive functioning: Category and Letter fluency and the Wisconsin Card Sorting Test (WCST) were used to evaluate executive functioning. ${ }^{17,19}$

6. Psychomotor function: Trail Making Test part A, Stroop Color Word Test parts A (word reading) and B (color naming), Simple and 2-choice reaction tasks evaluated psychomotor function. ${ }^{19}$ General health and quality of life were evaluated by means of the RAND-36 questionnaire ${ }^{23,24}$; the Profile of Mood States (POMS) determined depressive mood disorders. ${ }^{19}$ To explore whether measurement of cognitive speed was biased by physical impairments, we tested fine motor skills using the Grooved Pegboard. ${ }^{25}$

These tests are described in the Appendix.

Test results were expressed as standard $T$-scores corrected for age and education with use of the control group to form normative scores for the neuropsychological measures (mean score in control subjects, 50; standard deviation, 10; a higher score indicates better performance). ${ }^{26}$

\section{Data Analysis}

To examine relative differences between groups, we performed multivariate analyses of variance (MANOVAs) within each neuropsychological domain separately using $T$-scores corrected for age and education. If multivariate comparisons demonstrated significant results $(p<0.05$, Pillais' Trace two-tailed), $t$ tests with Bonferroni correction for the number of comparisons were performed to evaluate single neuropsychological measures.

As a next step, we tested for statistical interaction between treatment group (dexamethasone vs placebo) and the causative pathogen ( $S$. pneumoniae vs $N$. meningitidis) by adding 
multiplicative interaction terms to the multivariate comparisons. Statistically significant interactions within a cognitive domain were analyzed by linear regression with the individual neuropsychological measure as the dependent variable and the interaction term as the independent variable. The strength of the associations between treatment and test results within each patient group was expressed as regression coefficients (a positive regression coefficient indicates a higher score in the subgroup treated with adjunctive dexamethasone than in those receiving placebo).

A test score was categorized as impaired if at least two standard deviations below the mean score of the control group, after correction for age and education. Cognitive dysfunction was considered to be present if performance, reflected by the number of impaired test results, was worse than the fifth centile of the control group. To compare cognitive speed between groups, we formed a speed composite score for each participant by calculation of a mean $T$-score of tests in which cognitive speed is important, as described previously. ${ }^{5,27}$ Cognitive slowness was defined as a speed composite score worse than the fifth centile of the control group, after correction for age and education.

We hypothesized the existence of clinically significant cognitive impairment for patients who recovered from bacterial meningitis, with higher rates of cognitive impairment in patients receiving adjunctive dexamethasone than in those treated with placebo. ${ }^{2,10}$ In addition, we hypothesized higher rates of cognitive impairment in survivors of pneumococcal meningitis compared with those after meningococcal meningitis. ${ }^{5}$ For nonparametric testing, Mann-Whitney $U, \chi^{2}$, or Fisher's exact statistics were used. Data collected at the time of the original and follow-up studies were compared by the Wilcoxon signed rank test. A sample of size of 25 patients in each group is necessary to detect clinically relevant differences (effect size of 0.8 [defined as mean difference/standard deviation]); univariate power of 0.80 , and $\alpha=0.05$. For all comparisons, two-tailed $p$ values were used, and we considered $p<0.05$ to be statistically significant.

\section{Role of the Funding Source}

The funding source had no role in the study design; in the collection, analysis, and interpretation of data; in the writing of the report; or in the decision to submit this article for publication.

\section{Results}

In the European study, 32 of 301 patients (11\%) died within 8 weeks after admission. Of 269 surviving patients, 177 (66\%) had bacterial meningitis due to $S$. pneumoniae $(\mathrm{n}=83$ ) or $N$. meningitidis $(\mathrm{n}=94)$, as proved by CSF culture (Fig); 26 patients (15\%) could not be reached. In addition, exclusion criteria were met in 52 patients: death $(\mathrm{n}=19)$, residence outside the Netherlands $(\mathrm{n}=11)$, somatic or psychiatric illnesses

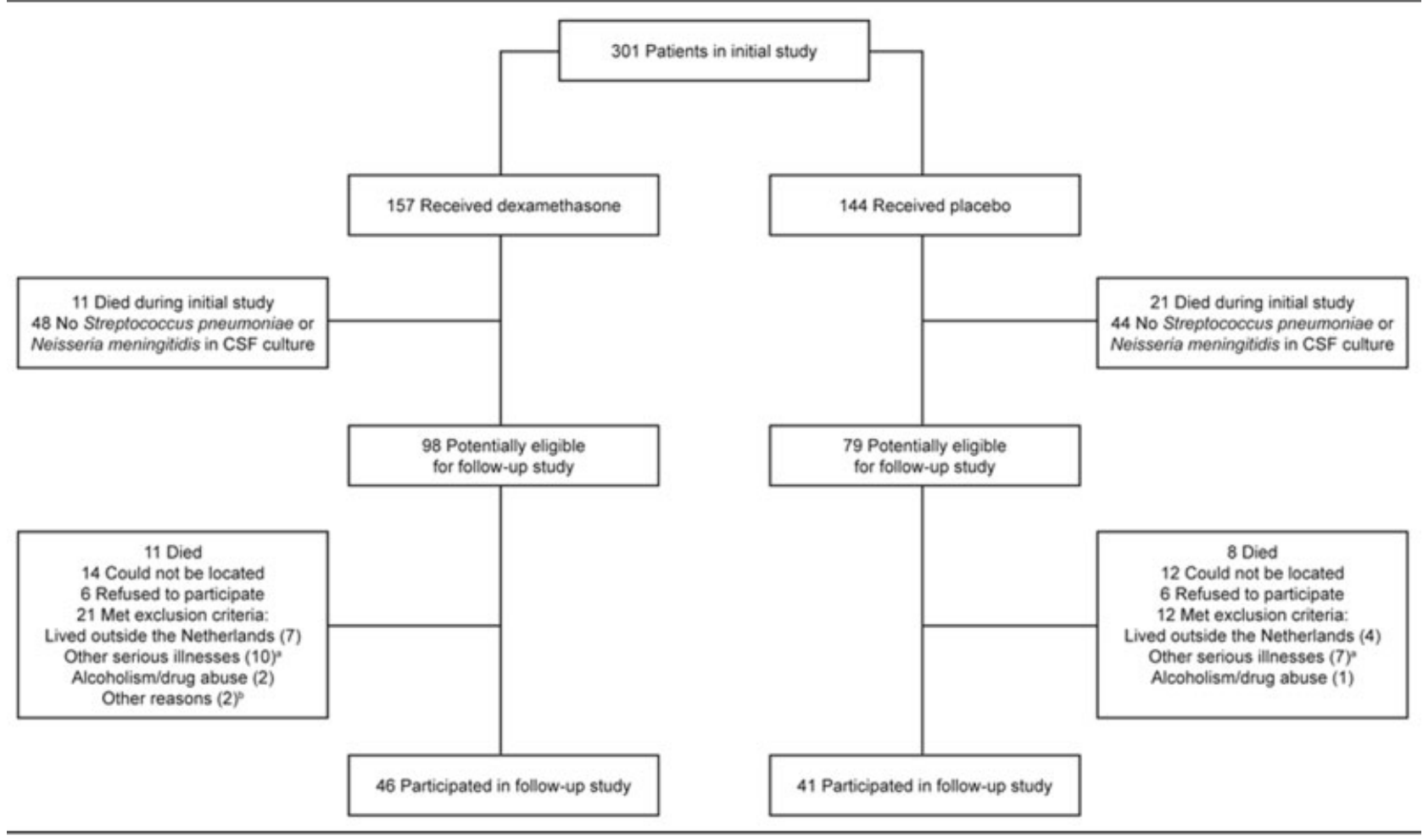

Fig. Patient selection in study of long-term outcome after bacterial meningitis. ${ }^{a}$ Systemic neoplasm $(n=4)$, psychiatric disorders $(n=4)$, severe bilateral deafness $(n=4)$, congenital deaf mutism $(n=1)$, mental retardation $(n=1)$, brain tumor $(n=1)$, rheumatoid arthritis $(n=1)$, bullet in the head $(n=1) .{ }^{b}$ One patient had insufficient mastery of Dutch language, and one patient was younger than 16 years. CSF = cerebrospinal fluid. 
not related to meningitis that impair cognitive testing $(\mathrm{n}=17)$, history of alcoholism $(\mathrm{n}=2)$ or other substance abuse $(\mathrm{n}=1)$, insufficient mastery of the Dutch language $(\mathrm{n}=1)$, or age younger than 16 years $(\mathrm{n}=$ 1; protocol violation of European study). In total, 99 patients were eligible for follow-up; of those eligible, 12 patients (12\%) refused to participate (all had a GOS score of 5, as assessed by telephonic interview). Thus, 87 of 99 patients $(88 \%)$ were included in this follow-up study. Demographic characteristics (age and sex), causative pathogens, and score on the GOS (8 weeks after discharge) were similar for eligible and participating patients. A total of 50 control subjects were recruited. Sex distribution (17 of 50 [34\%] control subjects were male; 43 of 87 [49\%] patients were male), age distribution (mean age, $49 \pm 15$ years for control subjects vs $47 \pm 16$ years for patients), and level of education $(13.2 \pm 1.6$ years for control subjects vs $12.9 \pm 2.3$ years for patients) were similar in patients and control subjects.

Clinical characteristics at time of admission were similar in the dexamethasone- and placebo-treated groups (Table 1). Focal neurological deficits were present in 23 of 87 patients (26\%), and 10 patients $(12 \%)$ were comatose on admission. CSF results demonstrated S. pneumoniae in 38 patients $(44 \%)$ and $N$. meningitidis in 49 (56\%); 46 patients $(53 \%)$ received adjunctive dexamethasone and $41(47 \%)$ received placebo. Eight weeks after discharge, focal neurological deficits were present in 12 of 87 patients (14\%). Outcome was graded as favorable in 80 of 87 patients (92\%); $6(7 \%)$ patients had a GOS score of 4 , and 1 patient $(1 \%)$ had a score of 3 .

Neurological examination was performed in all patients in this follow-up study. The median time between discharge and neurological examination was 99 months (range, 30-146 months) and was similar in both treatment groups. Neurological deficits were present in 17 of 87 patients (20\%); there were no differences between treatment groups (Table 2). Eighty patients $(92 \%)$ had a GOS score of 5, and $7(8 \%)$ had a GOS score of 4 . All patients with a GOS score of 4 had neurological sequelae; two patients had focal cerebral deficits, and the additional five patients had cranial nerve palsies. Ten patients $(11 \%)$ had only slight hearing impairment without significant interference in daily life: These patients also received a GOS score of 5. Most patients had similar scores on the GOS compared with the evaluation 8 weeks after admission $(p=0.76)$. Patients after pneumococcal meningitis had a higher rate of focal neurological deficits and were more likely to have a GOS score of 4 than those after meningococcal meningitis.

Audiological examination was performed in all participants who were able/willing to travel to our hospital: 51 of 87 patients (59\%) and 35 of 50 control subjects (70\%; Table 3). The degree of hearing loss in survivors of bacterial meningitis was similar to that in

Table 1. Characteristics of Patients in Study of Long-Term Outcome after Bacterial Meningitis

\begin{tabular}{|c|c|c|}
\hline Variable & $\begin{array}{l}\text { Dexamethasone Group } \\
\qquad(\mathrm{N}=46)\end{array}$ & $\begin{array}{l}\text { Placebo Group } \\
\quad(\mathrm{N}=41)\end{array}$ \\
\hline \multicolumn{3}{|l|}{ Characteristics on admission in initial study } \\
\hline Mean age at time of meningitis $( \pm S D), y r$ & $42 \pm 16$ & $38 \pm 18$ \\
\hline Alcoholism or substance abuse, $\mathrm{n}$ & 0 & 0 \\
\hline Sex: M/F & $19 / 27$ & $24 / 17$ \\
\hline Mean Glasgow Coma Scale score $( \pm S D)$ & $11 \pm 3$ & $11 \pm 3$ \\
\hline Coma, n (\%) & $6(13)$ & $4(10)$ \\
\hline Focal neurological abnormalities, n (\%) & $12(26)$ & $11(27)$ \\
\hline Focal cerebral deficits, ${ }^{\mathrm{a}} \mathrm{n}(\%)$ & $10(22)$ & $8(20)$ \\
\hline Cranial nerve palsies, n (\%) & $4(9)$ & $5(12)$ \\
\hline \multicolumn{3}{|l|}{ Laboratory results } \\
\hline Streptococcus pneumoniae in CSF culture, n (\%) & $22(48)$ & $16(39)$ \\
\hline Neisseria meningitidis in CSF culture, $\mathrm{n}(\%)$ & $24(52)$ & $25(61)$ \\
\hline Mean CSF leukocyte count $( \pm S D), 10^{3}$ cells $/ \mathrm{mm}^{3}$ & $23 \pm 26$ & $24 \pm 26$ \\
\hline Mean CSF protein level $( \pm S D), \mathrm{gm} / \mathrm{L}$ & $4.6 \pm 3.3$ & $5.2 \pm 3.3$ \\
\hline Mean CSF glucose level $( \pm S D), \mathrm{mg} / \mathrm{dl}$ & $1.3 \pm 1.7$ & $1.1 \pm 1.2$ \\
\hline Positive blood culture, n (\%) & $26 / 38(68)$ & $22 / 38(58)$ \\
\hline \multicolumn{3}{|l|}{ Neurological findings at discharge in initial study } \\
\hline GOS score 3, n (\%) & $1(2)$ & 0 \\
\hline GOS score $4, \mathrm{n}(\%)$ & $2(4)$ & $4(10)$ \\
\hline GOS score $5, \mathrm{n}(\%)$ & $43(94)$ & $37(90)$ \\
\hline Focal neurological abnormalities, n (\%) & $6(13)$ & $6(15)$ \\
\hline Focal cerebral deficits, ${ }^{a}$ n (\%) & $1(2)$ & $1(2)$ \\
\hline Cranial nerve palsies, n (\%) & $5(11)$ & $6(15)$ \\
\hline
\end{tabular}

${ }^{a}$ Defined as aphasia, monoparesis, or hemiparesis. SD = standard deviation; CSF = cerebrospinal fluid; GOS = Glasgow Outcome Scale. 


\begin{tabular}{lcccc}
\hline & $\begin{array}{c}\text { Dexamethasone } \\
\text { Group } \\
(\mathrm{N}=46)\end{array}$ & $\begin{array}{c}\text { Placebo Group } \\
(\mathrm{N}=41)\end{array}$ & $\begin{array}{c}\text { Pneumococcal } \\
\text { Meningitis } \\
\text { Patients } \\
(\mathrm{N}=38)\end{array}$ & $\begin{array}{c}\text { Meningococcal } \\
\text { Meningitis } \\
\text { Patients } \\
(\mathrm{N}=49)\end{array}$ \\
\hline $\begin{array}{l}\text { Focal neurological deficits, n (\%) } \\
\text { Focal cerebral deficits, }\end{array}$ & $10(22)$ & $7(17)$ & $13(34)$ & $4(8)^{\mathrm{a}}$ \\
Cranial nerve palsies, $\mathrm{n}(\%)$ & $1(2)$ & $1(2)$ & $2(5)$ & 0 \\
GOS score, 5:4 & $10(22)$ & $7(17)$ & $13(34)$ & $4(8)^{\mathrm{a}}$ \\
\hline
\end{tabular}

Comparison of pneumococcal versus meningococcal meningitis patients:

${ }^{\mathrm{a}} p=0.002, \chi^{2}$ test two-tailed;

${ }_{c}^{p} p=0.04$, Fisher's exact test two-tailed.

${ }^{\mathrm{b}}$ Slight hemiparesis in combination with hearing loss in both cases.

GOS = Glasgow Outcome Scale.

control subjects (median, 13 [range, 0-87] vs 10 [range, $2-35 \mathrm{~dB}$ ]; $p=0.41)$. The median degree of hearing loss was higher in adults surviving pneumococcal meningitis than in those after meningococcal meningitis (median, 18 [range, 2-87] vs 8 [range, $0-62 \mathrm{~dB}] ; p=0.01)$. The degree of hearing loss was similar in patients treated with dexamethasone and placebo (median, 13 [range, 0-72] vs 13 [range, $0-87 \mathrm{~dB}] ; p=0.76)$.

Neuropsychological examination was performed in all patients and control subjects. Demographic characteristics and interval between meningitis and testing for patients treated with dexamethasone and placebo were similar (Table 4). MANOVAs within neuropsychological domains showed no significant overall group differences between both treatment groups for "intelligence" $(p=0.06)$, "memory" ( $p=0.15)$, "executive functioning" ( $p=0.08)$, "attention" $(p=0.68)$, and "psychomotor speed" ( $p=0.72)$. Both treatment groups also had similar scores on questionnaires for general health and quality of life (RAND-36). Patients who received adjunctive dexamethasone had higher scores on the item "depression" than those with placebo $(p=0.04)$; results of the multivariate analyses for the cognitive domains remained similar after correction for depression scores.

The number of impaired test results for both treatment groups was similar as well (Table 5). Based on performance in the control group, a patient was considered to have cognitive dysfunction if he or she had three or more impaired test results. According to these criteria, cognitive dysfunction was present in 4 of the 46 (9\%) patients treated with dexamethasone compared with 7 of 41 patients in the placebo group (17\%; $p=0.24)$. Both treatment groups had similar results on the speed composite score $(p=0.14)$. Performance on the speed composite score showed no significant correlations with performance on the Grooved Pegboard and scores on the item "depression" of the POMS.

In patients with pneumococcal meningitis, demographic characteristics were similar in both treatment groups, although the interval between meningitis and testing was longer in those treated with dexamethasone $(p=0.04$; Table 6). MANOVAs within neuropsychological domains showed significant overall group differences between both treatment groups for "memory" $(p=0.004)$ and "executive functioning" ( $p=0.01)$,

Table 3. Results of Audiological Examinations in Control Subjects and Survivors of Bacterial Meningitis Treated with Adjunctive Dexamethasone or Placebo

\begin{tabular}{|c|c|c|c|c|c|}
\hline Variable & $\begin{array}{l}\text { Control } \\
\text { Subjects } \\
(\mathrm{N}=35)\end{array}$ & $\begin{array}{c}\text { Dexamethasone } \\
\text { Group } \\
(\mathrm{N}=24)\end{array}$ & $\begin{array}{l}\text { Placebo } \\
\text { Group } \\
(\mathrm{N}=27)\end{array}$ & $\begin{array}{l}\text { Pneumococcal } \\
\text { Meningitis } \\
\text { Patients } \\
(\mathrm{N}=25)\end{array}$ & $\begin{array}{c}\text { Meningococcal } \\
\text { Meningitis } \\
\text { Patients } \\
(\mathrm{N}=26)\end{array}$ \\
\hline \multicolumn{6}{|l|}{ Degree of pure-tone hearing loss, $\mathrm{n}(\%)$} \\
\hline$<30 \mathrm{~dB}$ & $32(91)$ & $18(75)$ & $20(74)$ & $16(64)$ & $22(85)$ \\
\hline$\geq 30-55 \mathrm{~dB}$ & $3(9)$ & $4(17)$ & $5(19)$ & $6(24)$ & $3(12)$ \\
\hline$\geq 55-70 \mathrm{~dB}$ & 0 & $1(4)$ & $1(4)$ & $1(4)$ & $1(4)$ \\
\hline$\geq 70-90 \mathrm{~dB}$ & 0 & $1(4)$ & $1(4)$ & $2(8)$ & 0 \\
\hline $\begin{array}{l}\text { Median speech recognition threshold } \\
\text { (range), } \mathrm{dB}\end{array}$ & $65(50-90)$ & $70(50-120)$ & $65(50-120)$ & $70(50-120)$ & $65(50-110)$ \\
\hline
\end{tabular}


Table 4. Comparison of Neuropsychological Test Results in Adults after Bacterial Meningitis Treated with Adjunctive Dexamethasone or Placebo

\begin{tabular}{|c|c|c|c|}
\hline Variable, Domain & $\begin{array}{l}\text { Dexamethasone Group } \\
\qquad(\mathrm{N}=46)\end{array}$ & $\begin{array}{l}\text { Placebo Group } \\
\quad(\mathrm{N}=41)\end{array}$ & $p^{\mathrm{a}}$ \\
\hline \multicolumn{4}{|l|}{ Demographic characteristics } \\
\hline Mean age at time of testing (SD), yr & $49.7(15.9)$ & $44.7(16.7)$ & 0.16 \\
\hline Mean education (SD), yr & $13.0(2.1)$ & $12.9(2.6)$ & 0.81 \\
\hline Median months from meningitis to testing (range) & $100.0(39-146)$ & $98.0(30-140)$ & $0.37^{\mathrm{b}}$ \\
\hline Mean premorbid intelligence (IQ) (SD) & $102.1(18.2)$ & $96.5(14.5)$ & 0.12 \\
\hline \multicolumn{4}{|l|}{ Mean intelligence score (SD): GIT } \\
\hline Intelligence (IQ) & $108.2(15.8)$ & $107.8(14.8)$ & 0.93 \\
\hline Verbal reasoning & $50.6(8.7)$ & $51.8(12.2)$ & 0.60 \\
\hline Visuospatial reasoning & $48.1(10.3)$ & $52.8(14.0)$ & 0.09 \\
\hline Numerical speed & $47.0(11.0)$ & $50.5(10.0)$ & 0.14 \\
\hline \multicolumn{4}{|l|}{ Mean memory score (SD) } \\
\hline Rey's AVLT immediate & $45.4(9.5)$ & $46.5(8.7)$ & 0.59 \\
\hline Rey's AVLT delayed & $47.2(8.2)$ & $46.4(9.6)$ & 0.65 \\
\hline Rey's AVLT recognition & $48.0(10.8)$ & $43.6(14.0)$ & 0.10 \\
\hline WMS-R immediate & $50.9(7.9)$ & $51.3(6.3)$ & 0.82 \\
\hline WMS-R delayed & $50.3(8.6)$ & $50.3(9.9)$ & 0.99 \\
\hline RBMT immediate & $50.4(8.8)$ & $50.9(11.3)$ & 0.80 \\
\hline RBMT delayed & $50.5(10.1)$ & $49.1(11.7)$ & 0.56 \\
\hline WAIS-R Digit Symbol Test & $47.6(9.8)$ & $50.4(9.2)$ & 0.20 \\
\hline Mean language score (SD): Boston Naming Test & $50.8(14.2)$ & $47.6(17.0)$ & 0.35 \\
\hline \multicolumn{4}{|l|}{ Mean attention score (SD) } \\
\hline Trail Making Test part B & $44.3(12.9)$ & $46.7(12.7)$ & 0.39 \\
\hline SCWT part C (interference condition) & $47.8(13.1)$ & $48.1(15.4)$ & 0.94 \\
\hline WAIS-R Digit Span Test & $47.6(9.8)$ & $50.4(9.2)$ & 0.35 \\
\hline \multicolumn{4}{|l|}{ Mean executive functioning score (SD) } \\
\hline Category fluency & $52.7(11.9)$ & $51.3(10.9)$ & 0.57 \\
\hline Letter fluency & $48.5(9.3)$ & $48.5(9.1)$ & 0.97 \\
\hline Number of WCST categories & $50.6(9.8)$ & $48.9(9.3)$ & 0.45 \\
\hline Number of WCST total errors & $53.1(9.2)$ & $47.4(11.6)$ & 0.02 \\
\hline Number of WCST perseverative errors & $49.5(8.9)$ & $45.1(12.1)$ & 0.07 \\
\hline \multicolumn{4}{|l|}{ Mean psychomotor speed score (SD) } \\
\hline Trail Making part A & $47.6(11.0)$ & $46.6(11.9)$ & 0.69 \\
\hline SCWT Test part A (word reading) & $47.6(11.3)$ & $48.4(10.3)$ & 0.74 \\
\hline SCWT part B (color naming) & $46.6(12.7)$ & $46.7(10.1)$ & 0.39 \\
\hline Visual reaction task, dominant hand & $47.1(10.2)$ & $48.5(10.2)$ & 0.54 \\
\hline Visual reaction task, nondominant hand & $47.2(11.9)$ & $50.0(8.5)$ & 0.23 \\
\hline Binary choice reaction task & $50.5(12.1)$ & $48.5(12.4)$ & 0.47 \\
\hline
\end{tabular}

Scores are expressed as $T$-scores corrected for age and education.

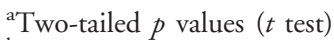

${ }^{\mathrm{b}}$ Two-tailed $p$ value of Mann-Whitney $U$ test.

$\mathrm{SD}=$ standard deviation; IQ = intelligence quotient; GIT = Groningen Intelligence Tests; AVLT = Auditory Verbal Learning Test; WMS$\mathrm{R}=$ Wechsler Memory Scale-Revised; RBMT = Rivermead Behavioural Memory Test; WAIS-R = Wechsler Adult Intelligence Scale-Revised; SCWT $=$ Stroop Color Word Test; WCST $=$ Wisconsin Card Sorting Test.

Table 5. Number of Impaired Tests in Control Subjects and Survivors of Bacterial Meningitis Treated with Adjunctive Dexamethasone or Placebo

\begin{tabular}{cccc}
\hline $\begin{array}{l}\text { Number } \\
\text { of Tests } \\
\text { Impaired }\end{array}$ & $\begin{array}{c}\text { Control } \\
\text { Subjects } \\
(\mathrm{N}=50)\end{array}$ & $\begin{array}{c}\text { Dexamethasone } \\
\text { Group } \\
(\mathrm{N}=46)\end{array}$ & $\begin{array}{c}\text { Placebo Group } \\
(\mathrm{N}=41)\end{array}$ \\
\hline 0 & $32(64 \%)$ & $21(46 \%)$ & $21(51 \%)$ \\
1 & $11(22 \%)$ & $12(26 \%)$ & $9(22 \%)$ \\
2 & $5(10 \%)$ & $9(20 \%)$ & $4(10 \%)$ \\
3 & $1(2 \%)$ & $3(7 \%)$ & $2(5 \%)$ \\
$\geq 4$ & $1(2 \%)$ & $1(2 \%)$ & $5(12 \%)$ \\
\hline
\end{tabular}

but not for "intelligence" ( $p=0.52)$, “attention" $(p=$ $0.68)$, and "psychomotor speed" $(p=0.86)$. Results of the multivariate analyses for the cognitive domains remained similar after correction for the interval between meningitis and testing. Pneumococcal meningitis patients who were treated with dexamethasone performed significantly better on the AVLT recognition than those treated with placebo $(p=0.004$, Bonferroni correction). Performance on the other neuropsychological tests within the domains of "memory" and "executive functioning" was similar in both treatment groups.

In patients with meningococcal meningitis, demo- 
Table 6. Comparison of Neuropsychological Test Results in Adults after Pneumococcal Meningitis Treated with Adjunctive Dexamethasone or Placebo

\begin{tabular}{|c|c|c|c|}
\hline Variable, Domain & $\begin{array}{l}\text { Dexamethasone Group } \\
\qquad(\mathrm{N}=22)\end{array}$ & $\begin{array}{l}\text { Placebo Group } \\
\quad(\mathrm{N}=16)\end{array}$ & $p^{\mathrm{a}}$ \\
\hline \multicolumn{4}{|l|}{ Demographic characteristics } \\
\hline Mean age at time of testing (SD), yr & $56.5(13.5)$ & $53.9(15.7)$ & 0.59 \\
\hline Mean education (SD), yr & $12.3(2.3)$ & $12.0(3.2)$ & 0.72 \\
\hline Median months from meningitis to testing (range) & $104(40-141)$ & $71.0(36-140)$ & $0.04^{\mathrm{b}}$ \\
\hline Mean premorbid intelligence (IQ) score & $101.0(17.4)$ & $99.2(17.9)$ & 0.75 \\
\hline \multicolumn{4}{|l|}{ Mean intelligence score (SD): GIT } \\
\hline Intelligence (IQ) & $106.7(15.5)$ & $105.6(15.8)$ & 0.84 \\
\hline Verbal reasoning & $48.8(8.6)$ & $51.8(12.3)$ & 0.39 \\
\hline Visuospatial reasoning & $48.7(11.9)$ & $54.5(13.9)$ & 0.20 \\
\hline Numerical speed & $44.5(10.7)$ & $46.4(9.8)$ & 0.59 \\
\hline \multicolumn{4}{|l|}{ Mean memory score (SD) } \\
\hline Rey's AVLT immediate & $45.2(8.9)$ & $41.1(8.6)$ & 0.16 \\
\hline Rey's AVLT delayed & $46.7(7.1)$ & $42.0(9.0)$ & 0.08 \\
\hline Rey's AVLT recognition & $49.4(11.6)$ & $36.6(13.6)$ & 0.0004 \\
\hline WMS-R immediate & $49.4(9.0)$ & $50.5(6.8)$ & 0.71 \\
\hline WMS-R delayed & $47.8(10.0)$ & $48.8(14.0)$ & 0.82 \\
\hline RBMT immediate & $50.5(6.6)$ & $51.2(11.3)$ & 0.82 \\
\hline RBMT delayed & $51.0(7.3)$ & $52.0(13.7)$ & 0.79 \\
\hline WAIS-R Digit Span Test & $48.1(9.7)$ & $53.3(11.4)$ & 0.16 \\
\hline Mean language score (SD): Boston Naming Test & $51.6(12.7)$ & $43.9(19.9)$ & 0.16 \\
\hline \multicolumn{4}{|l|}{ Mean attention score (SD) } \\
\hline Trail Making Test part B & $40.6(12.6)$ & $43.9(17.8)$ & 0.50 \\
\hline SCWT part C (interference condition) & $47.3(13.1)$ & $46.4(20.4)$ & 0.87 \\
\hline WAIS-R Digit Span Test & $48.1(9.7)$ & $53.3(11.4)$ & 0.16 \\
\hline \multicolumn{4}{|l|}{ Mean executive functioning score (SD) } \\
\hline Category fluency & $55.8(10.3)$ & $50.3(10.4)$ & 0.11 \\
\hline Letter fluency & $48.6(10.9)$ & $46.5(10.9)$ & 0.59 \\
\hline Number of WCST categories & $52.8(12.1)$ & $51.1(11.5)$ & 0.67 \\
\hline Number of WCST total errors & $54.1(11.4)$ & $46.7(14.0)$ & 0.09 \\
\hline Number of WCST perseverative errors & $49.1(11.7)$ & $44.2(14.7)$ & 0.28 \\
\hline \multicolumn{4}{|l|}{ Mean psychomotor speed score (SD) } \\
\hline Trail Making part A & $44.3(12.5)$ & $43.1(14.8)$ & 0.79 \\
\hline SCWT Test part A (word reading) & $47.9(13.7)$ & $48.4(9.8)$ & 0.91 \\
\hline SCWT part B (color naming) & $46.4(14.3)$ & $47.9(6.9)$ & 0.72 \\
\hline Visual reaction task, dominant hand & $44.5(12.9)$ & $47.8(12.7)$ & 0.46 \\
\hline Visual reaction task, nondominant hand & $45.9(15.1)$ & $49.0(11.9)$ & 0.52 \\
\hline Binary choice reaction task & $49.9(13.1)$ & $45.6(17.8)$ & 0.40 \\
\hline
\end{tabular}

Scores are expressed as $T$-scores corrected for age and education.

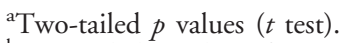

${ }^{\mathrm{b}}$ Two-tailed $p$ value of Mann-Whitney $U$ test.

$\mathrm{SD}=$ standard deviation; IQ $=$ intelligence quotient; GIT $=$ Groningen Intelligence Tests; AVLT = Auditory Verbal Learning Test; WMS$\mathrm{R}=$ Wechsler Memory Scale-Revised; RBMT = Rivermead Behavioural Memory Test; WAIS-R = Wechsler Adult Intelligence Scale-Revised; SCWT $=$ Stroop Color Word Test; WCST $=$ Wisconsin Card Sorting Test.

graphic characteristics in patients treated with dexamethasone and placebo were similar (Table 7). MANOVAs within neuropsychological domains showed no significant overall group differences between both treatment groups for "intelligence" $(p=$ $0.23)$, "memory" $(p=0.23)$, "executive functioning" $(p=0.50)$, "attention" $(p=0.99)$, and "psychomotor speed" $(p=0.32)$.

The interaction between the causative pathogen and treatment group was significant for the multivariate comparison of test results within the domain of "memory" $(p=0.007)$, but not within any of the other cognitive domains ( $p>0.55$ for all). In a linear regression analysis, this interaction was significant for performance on two subtests of the AVLT. Dexamethasone therapy was associated with higher scores on the AVLT immediate recall in patients after pneumococcal meningitis and with lower scores on the same test in survivors of meningococcal meningitis; regression coefficient in the subgroup of pneumococcal patients was 0.23 compared with -0.25 within meningococcal patients $(p$ interaction $=0.03)$. Similar associations were found for performance on the AVLT recognition (regression coefficient of 0.46 in pneumococcal patients 
Table 7. Comparison of Neuropsychological Test Results in Adults after Meningococcal Meningitis Treated with Adjunctive Dexamethasone or Placebo

\begin{tabular}{|c|c|c|c|}
\hline Variable, Domain & $\begin{array}{l}\text { Dexamethasone Group } \\
(\mathrm{N}=24)\end{array}$ & $\begin{array}{l}\text { Placebo Group } \\
(\mathrm{N}=25)\end{array}$ & $p^{\mathrm{a}}$ \\
\hline \multicolumn{4}{|l|}{ Demographic characteristics } \\
\hline Mean age at time of testing (SD), yr & $43.5(15.6)$ & $38.8(14.8)$ & 0.29 \\
\hline Mean education (SD), yr & $13.6(1.8)$ & $13.4(1.9)$ & 0.73 \\
\hline Median months from meningitis to testing (range) & $100.0(39-146)$ & $114.0(10-140)$ & $0.52^{\mathrm{b}}$ \\
\hline Mean premorbid intelligence (IQ) score (SD) & $103.1(19.3)$ & $94.8(12.1)$ & 0.08 \\
\hline \multicolumn{4}{|l|}{ Mean intelligence score (SD): GIT } \\
\hline Intelligence (IQ) & $109.5(16.2)$ & $109.1(14.4)$ & 0.94 \\
\hline Verbal reasoning & $52.3(8.6)$ & $51.8\left({ }^{\prime} \mathrm{r} 12.3\right)$ & 0.87 \\
\hline Visuospatial reasoning & $47.5(8.8)$ & $51.8(14.2)$ & 0.23 \\
\hline Numerical speed & $49.4(11.0)$ & $53.3(9.3)$ & 0.21 \\
\hline \multicolumn{4}{|l|}{ Mean memory score (SD) } \\
\hline Rey's AVLT immediate & $45.6(10.2)$ & $50.0(7.0)$ & 0.09 \\
\hline Rey's AVLT delayed & $47.7(9.3)$ & $49.2(9.0)$ & 0.58 \\
\hline Rey's AVLT recognition & $46.8(10.1)$ & $47.8(12.7)$ & 0.75 \\
\hline WMS-R immediate & $52.6(6.4)$ & $51.7(6.1)$ & 0.66 \\
\hline WMS-R delayed & $52.9(6.1)$ & $51.0(7.3)$ & 0.36 \\
\hline RBMT immediate & $50.2(10.5)$ & $50.7(11.4)$ & 0.45 \\
\hline RBMT delayed & $50.0(12.3)$ & $47.5(10.4)$ & 0.87 \\
\hline WAIS-R Digit Span Test & $47.2(10.1)$ & $48.7(7.3)$ & 0.57 \\
\hline Mean language score (SD): Boston Naming Test & $50.1(15.6)$ & $50.1(14.5)$ & 0.99 \\
\hline \multicolumn{4}{|l|}{ Mean attention score (SD) } \\
\hline Trail Making Test part B & $47.8(12.5)$ & $48.5(7.9)$ & 0.83 \\
\hline SCWT part C (interference condition) & $48.3(13.3)$ & $49.1(11.8)$ & 0.84 \\
\hline WAIS-R Digit Span Test & $47.2(10.1)$ & $48.7(7.3)$ & 0.57 \\
\hline \multicolumn{4}{|l|}{ Mean executive functioning score (SD) } \\
\hline Category fluency & $49.8(12.8)$ & $51.8(11.4)$ & 0.55 \\
\hline Letter fluency & $48.5(7.9)$ & $49.7(7.7)$ & 0.61 \\
\hline Number of WCST categories & $48.4(6.4)$ & $47.6(7.5)$ & 0.71 \\
\hline Number of WCST total errors & $52.0(6.5)$ & $47.8(10.2)$ & 0.12 \\
\hline Number of WCST perseverative errors & $49.8(5.0)$ & $45.7(10.5)$ & 0.11 \\
\hline \multicolumn{4}{|l|}{ Mean psychomotor speed score (SD) } \\
\hline Trail Making part A & $50.7(8.4)$ & $48.9(9.4)$ & 0.47 \\
\hline SCWT Test part A (word reading) & $47.3(8.4)$ & $48.4(10.9)$ & 0.70 \\
\hline SCWT part B (color naming) & $46.7(11.1)$ & $46.0(11.7)$ & 0.85 \\
\hline Visual reaction task, dominant hand & $49.4(6.3)$ & $48.9(8.5)$ & 0.83 \\
\hline Visual reaction task, nondominant hand & $48.3(8.2)$ & $50.7(5.5)$ & 0.26 \\
\hline Binary choice reaction task & $51.0(11.3)$ & 50.5 (6.9) & 0.85 \\
\hline
\end{tabular}

Scores are expressed as $T$-scores corrected for age and education.

${ }^{\text {a Two-tailed }} p$ values $(t$ test).

${ }^{\mathrm{b}}$ Two-tailed $p$ value of Mann-Whitney $U$ test.

$\mathrm{SD}=$ standard deviation; IQ $=$ intelligence quotient; GIT $=$ Groningen Intelligence Tests; AVLT = Auditory Verbal Learning Test; WMS$\mathrm{R}=$ Wechsler Memory Scale-Revised; RBMT = Rivermead Behavioural Memory Test; WAIS-R = Wechsler Adult Intelligence Scale-Revised; SCWT $=$ Stroop Color Word Test; WCST $=$ Wisconsin Card Sorting Test.

vs -0.05 in meningococcal patients; $p$ interaction $=$ $0.01)$.

Control subjects and patients had similar scores on premorbid intelligence $(99.6 \pm 18$ in control subjects vs $99.5 \pm 17$ in patients; $p=0.96$ ). MANOVAs showed no significant differences between patients and control subjects for "intelligence" ( $p=0.37)$, "memory" $(p=0.31)$, "executive functioning" $(p=0.06)$, "attention" ( $p=0.15)$, and "psychomotor speed" $(p=0.38)$. Both groups had similar scores on the RAND-36, the POMS, and the Grooved Pegboard.

Patients after pneumococcal meningitis were older $(p<0.0001)$ and had a lower level of education $(p=$ $0.01)$ than those after meningococcal meningitis (Table 8). Sex distribution in both groups was similar. MANOVAs showed significant overall group differences between survivors of pneumococcal meningitis and meningococcal meningitis for "memory" $(p=$ $0.01)$, but not for "intelligence" ( $p=0.12)$, "executive functioning" ( $p=0.08)$, "attention" $(p=0.12)$, and "psychomotor speed" ( $p=0.22)$. Patients after pneumococcal meningitis performed worse than those after meningococcal meningitis on all tasks of the AVLT and the Wechsler Memory Scale-Revised. These differ- 
Table 8. Neuropsychological Test Results in 87 Adults after Recovery from Bacterial Meningitis

\begin{tabular}{|c|c|c|c|}
\hline Variable, Domain & $\begin{array}{l}\text { Meningococcal Patients } \\
\qquad(\mathrm{N}=49)\end{array}$ & $\begin{array}{l}\text { Pneumococcal Patients } \\
\qquad(\mathrm{N}=38)\end{array}$ & $p^{\mathrm{a}}$ \\
\hline \multicolumn{4}{|l|}{ Demographic characteristics } \\
\hline Mean age at time of testing (SD), yr & $41.1(15.2)$ & $55.4(14.3)$ & $<0.0001$ \\
\hline Mean education (SD), yr & $13.5(1.8)$ & $12.2(2.7)$ & 0.01 \\
\hline Median months from meningitis to testing (range) & $110(30-146)$ & $88.5(36-141)$ & $0.20^{\mathrm{b}}$ \\
\hline Mean premorbid intelligence (IQ) score (SD) & $98.8(16.3)$ & $100.3(17.4)$ & 0.68 \\
\hline \multicolumn{4}{|l|}{ Mean intelligence score (SD): GIT } \\
\hline Intelligence (IQ) & $109.3(15.1)$ & $106.3(15.4)$ & 0.38 \\
\hline Verbal reasoning & $52.1(10.6)$ & $50.0(10.1)$ & 0.36 \\
\hline Visuospatial reasoning & $49.8(12.0)$ & $51.1(12.9)$ & 0.64 \\
\hline Numerical speed & $51.5(10.2)$ & $45.3(10.2)$ & 0.01 \\
\hline \multicolumn{4}{|l|}{ Mean memory score (SD) } \\
\hline Rey's AVLT immediate & $47.8(8.9)$ & $43.5(8.9)$ & 0.03 \\
\hline Rey's AVLT delayed & $48.5(9.1)$ & $44.7(8.2)$ & 0.05 \\
\hline Rey's AVLT recognition & $47.3(11.4)$ & $44.2(13.9)$ & 0.26 \\
\hline WMS-R immediate & $52.1(6.2)$ & $49.8(8.1)$ & 0.15 \\
\hline WMS-R delayed & $51.8(6.8)$ & $48.1(11.4)$ & 0.08 \\
\hline RBMT immediate & $50.5(10.9)$ & $50.8(8.6)$ & 0.91 \\
\hline RBMT delayed & $48.7(11.3)$ & $51.4(10.1)$ & 0.26 \\
\hline WAIS-R Digit Symbol Test & $47.9(8.7)$ & $50.2(10.6)$ & 0.29 \\
\hline Mean language score (SD): Boston Naming Test & $50.1(14.9)$ & $48.2(16.4)$ & 0.59 \\
\hline \multicolumn{4}{|l|}{ Mean attention score (SD) } \\
\hline Trail Making Test part B & $48.2(10.3)$ & $42.0(14.9)$ & 0.03 \\
\hline SCWT part C (interference condition) & $48.7(12.4)$ & $47.0(16.2)$ & 0.57 \\
\hline WAIS-R Digit Span Test & $47.9(8.7)$ & $50.2(10.6)$ & 0.29 \\
\hline \multicolumn{4}{|l|}{ Mean executive functioning score (SD) } \\
\hline Category fluency & $50.8(12.0)$ & $53.5(10.5)$ & 0.28 \\
\hline Letter fluency & $49.1(7.8)$ & $47.7(10.8)$ & 0.50 \\
\hline Number of WCST categories & $48.0(6.9)$ & $52.1(11.7)$ & 0.06 \\
\hline Number of WCST total errors & $49.9(8.7)$ & $51.2(12.8)$ & 0.61 \\
\hline Number of WCST perseverative errors & $47.7(8.4)$ & $47.1(13.0)$ & 0.82 \\
\hline \multicolumn{4}{|l|}{ Mean psychomotor speed score (SD) } \\
\hline Trail Making part A & $49.8(8.9)$ & $43.8(13.3)$ & 0.01 \\
\hline SCWT Test part A (word reading) & $47.8(9.7)$ & $48.1(12.1)$ & 0.93 \\
\hline SCWT part B (color naming) & $46.3(11.3)$ & $47.0(11.8)$ & 0.79 \\
\hline Visual reaction task, dominant hand & $49.2(7.4)$ & $45.9(12.8)$ & 0.15 \\
\hline Visual reaction task, nondominant hand & $49.5(7.0)$ & $47.2(13.7)$ & 0.33 \\
\hline Binary choice reaction task & $50.7(9.3)$ & $48.1(15.1)$ & 0.34 \\
\hline
\end{tabular}

Scores are expressed as $T$-scores correczay2354.tifted for age and education.

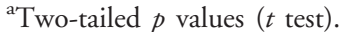

${ }^{\mathrm{b}}$ Two-tailed $p$ value of Mann-Whitney $U$ test.

$\mathrm{SD}=$ standard deviation; IQ = intelligence quotient; GIT = Groningen Intelligence Tests; AVLT = Auditory Verbal Learning Test; WMS$\mathrm{R}=$ Wechsler Memory Scale-Revised; RBMT = Rivermead Behavioural Memory Test; WAIS-R = Wechsler Adult Intelligence Scale-Revised; SCWT $=$ Stroop Color Word Test; WCST $=$ Wisconsin Card Sorting Test.

ences remained similar after correction for score on the speed composite measure, but they were no longer significant after Bonferroni correction for the number of comparisons. Patients who had pneumococcal meningitis experienced more impairment of everyday functioning due to physical problems (RAND-36; $p=$ 0.05). Both groups had similar scores on the Grooved Pegboard and the item "depression" of the POMS. The proportion of cognitive dysfunction was higher in patients after pneumococcal meningitis (8 of 38 [21\%] vs 3 of $49[6 \%] ; p=0.05$ ). Survivors of pneumococcal and meningococcal meningitis had similar results on the speed composite score $(p=0.09)$. Performance on the speed composite score was significantly associated with current intelligence in a linear regression analysis (intelligence quotient; $p<0.0001$, two-tailed), but not with the causative pathogen $(p=0.10)$, time since meningitis $(p=0.67)$, dexamethasone therapy $(p=$ $0.44)$, and GOS score $(p=0.86)$.

Quality-of-life questionnaires demonstrated that patients who were defined as cognitively impaired had higher scores on the item "depression" of the POMS $(p=0.003)$ and had lower scores on the RAND-36 on the subscales of social functioning $(p=0.04)$, mental health $(p=0.004)$, and general health $(p=$ 0.05). 


\section{Discussion}

Our results show that treatment with adjunctive dexamethasone is not associated with an increased risk for long-term cognitive impairment in adults with bacterial meningitis. Because adjunctive dexamethasone has become routine therapy in adults with suspected bacterial meningitis, this is important information for patients and their treating physicians. Our prospective study comprises the largest number of patients with longterm neurological and neuropsychological evaluation after bacterial meningitis to date and has enough power to detect relevant differences between the dexamethasone and placebo groups.

Treatment with adjunctive dexamethasone is associated with a reduced risk for an unfavorable outcome, including mortality, in adults with bacterial meningitis. ${ }^{6,7}$ Although treatment with adjunctive dexamethasone was not associated with apparent side effects in the European study, concerns existed about the effects on long-term cognitive outcome. ${ }^{13}$ In vitro studies have shown that corticosteroids are toxic to cultured hippocampal and cortical neurons. ${ }^{28}$ Animal studies demonstrated that striatal and hippocampal neurons are particularly vulnerable to dexamethasone. ${ }^{29,30}$ In addition, dexamethasone therapy increased hippocampal apoptosis and learning deficiencies in experimental bacterial meningitis. ${ }^{10,31}$ The dosage of dexamethasone used in these experimental studies and the European study were similar $(0.7 \mathrm{mg} / \mathrm{kg} /$ day $) .{ }^{10}$ Corticosteroidinduced damage of hippocampal neurons may account for some of the cognitive deficits that have been noted in healthy volunteers. ${ }^{29,30}$ In a study on long-term effects of early postnatal dexamethasone therapy for lung disease of prematurity, steroids were associated with impairments in neuromotor and cognitive function at school age. ${ }^{32}$ Our analyses showed no significant group differences between patients treated with adjunctive dexamethasone and placebo within any of the cognitive domains. Therefore, treatment with adjunctive dexamethasone does not worsen long-term cognitive outcome in adults after bacterial meningitis.

We found that $13 \%$ of the patients who survived bacterial meningitis had cognitive impairment. These results are identical to those of our previous study in which neuropsychological testing was performed in 51 adults with good recovery after bacterial meningitis. ${ }^{5} \mathrm{~A}$ recent German study evaluating cognitive outcome 1 to 12 years after bacterial meningitis found a much higher rate of cognitive impairment (37\%). ${ }^{33}$ These differences are explained by different criteria for cognitive dysfunction. The German study used less strict criteria, resulting in impaired cognitive functioning of the control subjects ranging from 7 to $27 \%$ per domain. If our criteria for cognitive dysfunction (score worse than the fifth centile of control group) were applied to the
German study, $10 \%$ of the patients in their study would have been rated as cognitively impaired. ${ }^{33}$

Patients after pneumococcal meningitis are at high risk for cognitive impairment. Cognitive impairment was found in $21 \%$ of the patients who survived pneumococcal meningitis. Patients after meningococcal meningitis had scores similar to those of the control subjects. These findings agree with our previous study that found cognitive impairment in $27 \%$ of the adults who made a good recovery after pneumococcal meningitis. ${ }^{5}$ The recent German study found no differences in cognitive outcome between survivors of pneumococcal and meningococcal meningitis ${ }^{33}$; however, these discordant findings may well be explained by the limited number of patients in the latter study. ${ }^{11}$

The main cognitive impairment in patients after pneumococcal meningitis consisted of memory deficits. Previous studies on cognitive outcome in adults who survived bacterial meningitis described cognitive slowness, impairment of psychomotor and visuoconstructive performance, and higher scores on depression scales. ${ }^{5,27,33-35}$ Our findings agree with previous observations. ${ }^{5,27,33}$ Hippocampal damage has been related to learning and memory deficits in humans and in animal models. ${ }^{10}$ This suggests that neuronal apoptosis in the hippocampal dentate gyrus represents the major neuropathological process responsible for cognitive impairment in survivors of bacterial meningitis.

Cognitive speed was not related to time since meningitis. This is in contrast with our previous study, which suggested that loss of cognitive speed might be partially reversible in time. ${ }^{27}$ This might well be explained by the longer time interval between meningitis and neuropsychological evaluation in this study compared with those previous studies (median, 8 years in this study [range, 30 months to 12 years] vs 14 months [range, 7-25 months] ${ }^{5}$ and 17 months [range, 6 months to 4 years], ${ }^{27}$ respectively). Our findings imply that neuropsychological impairment improves in the first years after bacterial meningitis and becomes relatively stable with time.

The association of adjunctive dexamethasone therapy with test results within the domain of memory differed between patients after pneumococcal and meningococcal meningitis. Whereas patients after pneumococcal meningitis that received steroid therapy performed slightly better on several memory-dependent tasks than those in the placebo group, our analyses showed a reverse association in the subgroup of meningococcal patients. Although these findings may have been due to chance, they are in line with the conflicting results of steroids on neuronal injury in experimental meningitis studies. ${ }^{10,12,36}$ Animal models of bacterial meningitis showed that a more severe inflammatory response within the subarachnoid space is associated with an increased risk for adverse outcome and neurological se- 
quelae. ${ }^{37}$ Although antiinflammatory treatment with corticosteroids can reduce the severity of CSF inflammation, dexamethasone increased hippocampal apoptosis in infant rats with pneumococcal meningitis. ${ }^{10}$ In contrast with these findings, adjunctive dexamethasone reduced acute brain injury and attenuated meningitisinduced memory deficits in mature rats after recovery from meningitis with group B Streptococcus. ${ }^{36}$ Because the inflammatory response and apoptotic pathways are both related to meningitis-induced brain damage, ${ }^{38}$ the lack of a negative effect of steroid therapy on cognitive outcome in this study may reflect potential steroidinduced brain injury being offset by the beneficial effects of inflammation or other not-yet-identified beneficial effects. Therefore, the effect of adjunctive dexamethasone on neuronal injury and cognitive outcome in bacterial meningitis may, in part, depend on the causative pathogen, the patient's age, and the severity of infection.

The most important limitation of our study was selection bias. Because our study evaluated patients who were previously included in a randomized, controlled trial, all patients met specific inclusion criteria. Baseline characteristics of patients in the European Dexamethasone Study were similar to those included in our nation-wide cohort of adults with culture-proved bacterial meningitis. ${ }^{1}$ Therefore, patients included in the European study are likely to be a representative sample of adults with bacterial meningitis. Of the potentially eligible patients in this study, $15 \%$ could not be contacted for participation in this study. This relatively high rate is probably due to the long time interval to the start of this follow-up study (11 years) and the anonymous inclusion of patients in the initial study. Although demographic characteristics and the score on the GOS 8 weeks after discharge in these lost-tofollow-up patients were similar to the other patients, this lack of follow-up data may have confounded our results.

In conclusion, we found that treatment with adjunctive dexamethasone is not associated with an increased risk for long-term cognitive impairment. In addition, our results show that adults who survive pneumococcal meningitis are at significant risk for long-term cognitive sequelae. Neuropsychological assessment can be useful as an additional outcome measure in studies on neurological infectious diseases.

\section{Appendix}

\section{Neuropsychological Tests and Questionnaires}

Boston Naming Test (short version): The short version of this test consists of a visual picture naming task in which 30 drawings are shown. These pictures are presented in order of difficulty. Score is number of correctly named pictures.
Dutch Adult Reading Test (DART): Fifty words with irregular spelling have to be read aloud. The number of correctly read words is transformed into an estimate of verbal intelligence. The DART is the Dutch counterpart of the National Adult Reading Test (NART). This test gives an estimate of premorbid verbal intelligence, because it correlates highly with other tests of verbal intelligence, although it is relatively insensitive to cognitive deterioration due to neurological disorders. Scores are intelligence quotients (population mean, 100; standard deviation, 15).

Groningen Intelligence Test (GIT), subtest Verbal Reasoning: The subject has to choose one word from five possibilities that correctly completes a $3 \times 2$ matrix of logical semantic relations (eg, black-white, high-low, hot-?).

GIT, subtest Visuospatial Reasoning: The subject has to decide which of several smaller geometric shapes are needed to fill a larger geometric figure (in a way similar to the oriental game of Tangram, but without actually manipulating the shapes).

GIT, subtest Numerical Speed: The subject completes as many written additions as possible in 1 minute.

Rey's Auditory Verbal Learning Test (AVLT): The subject has to memorize a series of 15 words in 5 learning trials. The test consists of an immediate recall task, a delayed recall task, and a recognition task.

Subtest Visual Reproduction of the Wechsler Memory Scale-Revised (WMS-R): Four geometric figures of increasing complexity are shown to the subject for 10 seconds each. Immediately after presentation of each figure and after a 30-minute delay, the subject is asked to draw the figures from memory. The quality of the drawings is scored by use of 41 criteria, resulting in a raw score range of 0 to 41 .

Stroop Color Word Test (SCWT): This test measures perceptual interference, response inhibition, and selective attention by having the subject read the names of 100 colors printed on a card (card 1), name the color of ink of colored squares (card 2), and name the color of ink of 100 nonmatching color words (card 3). Score is time to completion in seconds.

Subtest Story Recall of the Rivermead Behavioural Memory Test (RMBT): Two 21-item news messages are read to the subject, who repeats as many items as he or she can remember. After a 15-minute interval, the subject is asked to recall the message again.

Trail Making Test: The task is to connect consecutive numbers (part $A$ ) or numbers alternating with letters (part B) on a sheet of paper. This is a test of visual scanning, visuomotor and conceptual tracking, mental flexibility, and motor speed. 
Wisconsin Card Sorting Test (WCST): This test uses a deck of cards on which different numbers of different forms in different colors are shown. The task is to sort the cards according to one of three possible sorting rules (color, number, or form). These rules are not told but must be determined by the subject. After each sort, feedback is given on whether it was correct. Once a sorting rule has been found (10 correct sorts on a row), the subject has to change to a different rule. Of particular interest are perseverative errors of the kind where the subject keeps sorting according to a previously correct rule or to a rule that was wrong in the immediately preceding sort. The WCST is a test of concept formation and set shifting. Scores used in this study are the raw numbers of errors and perseverations.

GIT, Category Fluency: For 1 minute, the subject has to say as many words that belong to a particular semantic category. Two trials were done (animals and occupations).

Letter Fluency (Controlled Oral Word Association Test [COWAT]): For 1 minute, the subject has to say as many words that begin with a given letter. Three trials with different letters were done.

Simple reaction speed: Simple reaction speed was tested for the dominant and nondominant hands separately with use of visual stimuli. Scores are median reaction times in milliseconds.

Two-choice reaction speed: Two-choice reaction speed was tested with use of both hands and visual stimuli. Scores are median reaction times in milliseconds.

Grooved Pegboard: Fine motor skills and visual motor coordination were tested for the dominant and nondominant hands separately with use of a board with 25 holes with randomly positioned slots in which cones have to be inserted. Score is time to completion in seconds.

Subtest Digit Span of Wechsler Adult Intelligence ScaleRevised (WAIS-R): Subjects have to listen to increasingly longer lists of digits and to produce immediate recall in the actual and in reverse order from initial presentation. This test is a measure of attention, as well as working memory.

RAND-36: The RAND-36 is a questionnaire of general health and quality of life. It has been standardized for the Netherlands on a sample of subjects ( $\mathrm{n}=$ $1,063)$ that is roughly comparable with the present groups with respect to age and education.

Profile of Mood States (POMS): During the test session, an abbreviated version of the POMS was filled out by the patient. This is a list of 32 adjectives clustered in 5 subscales (depression, anger, fatigue, vigor, and tension) by which subjects describe their mood during the preceding week.

This study was supported by the Meningitis Research Foundation, United Kingdom (03/03, D.v.d.B.). The European Dexamethasone in Adulthood Bacterial Meningitis Study was supported NV Organon, which also supplied the study medication.

We are indebted to M. Neerings and M. Boymans for their help in the audiological examinations and to many physicians in the Netherlands for their cooperation.

\section{References}

1. van de Beek D, de Gans J, Spanjaard L, et al. Clinical features and prognostic factors in adults with bacterial meningitis. N Engl J Med 2004;351:1849-1859.

2. van de Beek D, de Gans J, Tunkel AR, Wijdicks EF. Community-acquired bacterial meningitis in adults. N Engl J Med 2006;354:44-53.

3. Weisfelt M, van de Beek D, Spanjaard L, et al. Clinical features, complications, and outcome in adults with pneumococcal meningitis: a prospective case series. Lancet Neurol 2006;5: 123-129.

4. Durand ML, Calderwood SB, Weber DJ, et al. Acute bacterial meningitis in adults. A review of 493 episodes. N Engl J Med 1993;328:21-28.

5. van de Beek D, Schmand B, de Gans J, et al. Cognitive impairment in adults with good recovery after bacterial meningitis. J Infect Dis 2002;186:1047-1052.

6. de Gans J, van de Beek D. Dexamethasone in adults with bacterial meningitis. N Engl J Med 2002;347:1549-1556.

7. van de Beek D, de Gans J, McIntyre P, Prasad K. Steroids in adults with acute bacterial meningitis: a systematic review. Lancet Infect Dis 2004;4:139-143.

8. Tunkel AR, Hartman BJ, Kaplan SL, et al. Practice guidelines for the management of bacterial meningitis. Clin Infect Dis 2004;39:1267-1284.

9. Sapolsky RM, Pulsinelli WA. Glucocorticoids potentiate ischemic injury to neurons: therapeutic implications. Science 1985; 229:1397-1400.

10. Leib SL, Heimgartner C, Bifrare YD, et al. Dexamethasone aggravates hippocampal apoptosis and learning deficiency in pneumococcal meningitis in infant rats. Pediatr Res 2003;54: 353-357.

11. van de Beek D, Weisfelt M, Hoogman M, et al. Neuropsychological sequelae of bacterial meningitis: alcoholism and adjunctive dexamethasone therapy. Brain 2006;129:E46.

12. Weisfelt M, van de Beek D, de Haan RJ, de Gans J. Dexamethasone and prognostic factors in adults with bacterial meningitis. J Neurol 2006;253:570-573.

13. Weisfelt M, de Gans J, van der Poll T, van de Beek D. Pneumococcal meningitis in adults: new approaches to management and prevention. Lancet Neurol 2006;5:332-342.

14. Kastenbauer S. Pneumococcal meningitis: a 21 st century perspective. Lancet Neurol 2006;5:104-105.

15. Jennet B, Bond M. Assessment of outcome after severe brain damage. Lancet 1975;1:480-484.

16. Dodge PR, Davis H, Feigin RD, et al. Prospective evaluation of hearing impairment as a sequela of acute bacterial meningitis. N Engl J Med 1984;311:869-874.

17. Luteijn F, van der Ploeg F. Groningen intelligentie test. Lisse, the Netherlands: Swets and Zeitlinger, 1983.

18. Schmand B, Lindeboom J, van Harskamp F. De Nederlandse leestest voor volwassenen. Lisse, the Netherlands: Swets and Zeitlinger, 1992. 
19. Lezak MD, Howieson BD, Loring DW. Neuropsychological assessment. 4th ed. New York: Oxford University Press, 2004.

20. Wilson B, Cockburn J, Baddeley A. Rivermead behavioural memory test. Oxford: Thames Valley Test Company, 1985.

21. Wechsler D. Wechsler Adult Intelligence Scale-Revised WAIS-R: test manual. New York: Psychological Corporation, 1981.

22. Kaplan E, Goodglass H, Weintraub S. Boston Naming Test. Philadelphia: Lea \& Febiger, 1983.

23. Brazier JE, Harper R, Jones NM, et al. Validating the SF-36 health survey questionnaire: new outcome measure for primary care. BMJ 1992;305:160-164.

24. van der Zee K, Sanderman R. Het meten van de algemene gezondheidstoestand met de RAND-36. Groningen, the Netherlands: Groningen University, 1993.

25. Baser CA, Ruff RM. Construct validity of the San Diego Neuropsychological Test Battery. Arch Clin Neuropsychol 1987;2: 13-32.

26. Mitrushina MN, Boone KB, D'Eli L. Handbook of normative data for neuropsychological assessment. New York: Oxford University Press, 1999.

27. Weisfelt M, van de Beek D, Hoogman M, et al. Cognitive outcome in adults with moderate disability after pneumococcal meningitis. J Infect 2006;52:433-439.

28. McIntosh LJ, Sapolsky RM. Glucocorticoids increase the accumulation of reactive oxygen species and enhance adriamycininduced toxicity in neuronal culture. Exp Neurol 1996;141: 201-206.

29. Mitchell IJ, Cooper AJ, Griffiths MR, Barber DJ. Phencyclidine and corticosteroids induce apoptosis of a subpopulation of striatal neurons: a neural substrate for psychosis? Neuroscience 1998;84:489-501.
30. Haynes LE, Griffiths MR, Hyde RE, et al. Dexamethasone induces limited apoptosis and extensive sublethal damage to specific subregions of the striatum and hippocampus: implications for mood disorders. Neuroscience 2001;104:57-69.

31. Zysk G, Bruck W, Gerber J, et al. Anti-inflammatory treatment influences neuronal apoptotic cell death in the dentate gyrus in experimental pneumococcal meningitis. J Neuropathol Exp Neurol 1996;55:722-728.

32. Yeh TF, Lin YJ, Lin HC, et al. Outcomes at school age after postnatal dexamethasone therapy for lung disease of prematurity. N Engl J Med 2004;350:1304-1313.

33. Schmidt H, Heimann B, Djukic M, et al. Neuropsychological sequelae of bacterial and viral meningitis. Brain 2006;129: 333-345.

34. Merkelbach S, Sittinger H, Schweizer I, Muller M. Cognitive outcome after bacterial meningitis. Acta Neurol Scand 2000; 102:118-123.

35. Bohr V, Rasmussen N, Hansen B, et al. Pneumococcal meningitis: an evaluation of prognostic factors in 164 cases based on mortality and on a study of lasting sequelae. J Infect 1985;10:143-157.

36. Irazuzta J, Pretzlaff RK, Courten-Myers G, et al. Dexamethasone decreases neurological sequelae and caspase activity. Intensive Care Med 2005;31:146-150.

37. Mustafa MM, Ramilo O, Mertsola J, et al. Modulation of inflammation and cachectin activity in relation to treatment of experimental Haemophilus influenzae type b meningitis. J Infect Dis $1989 ; 160: 818-825$.

38. Martins MR, Barichello T, Ritter C, et al. Dexamethasone therapy and memory performance. Intensive Care Med 2005;31: 1001. 